\title{
Design of Experimental Platform for Intelligent Car
}

\author{
Hongtao $\mathrm{Yu}^{1, \mathrm{a}^{*}}$, Sen Wang ${ }^{1, \mathrm{~b}}$, Heyan Wang ${ }^{2, \mathrm{c}}$ and Yanhua Lei ${ }^{1, \mathrm{~d}}$ \\ ${ }^{1}$ College of Automation, Shenyang Institute of Engineering, Shenyang Liaoning110136, China \\ ${ }^{2}$ Assets Distribution Department, Metering Center of the State Grid Liaoning Electric Power Supply \\ Co., Ltd, Shenyang Liaoning 110168, China \\ aneu970773@sohu.com, bwangs@sie.edu.cn, cwanghy@162.com, deiyh@sie.edu.cn
}

\section{Keywords: Experimental platform; Intelligent car; LabVIEW; PID; Virtual instrument}

\begin{abstract}
Based on the study of the principle of intelligent car movement, this paper designs the tracking process, the PID adjustment process, the real-time monitoring process, and displays the simulation interface on the intelligent car software platform. So that the simulation results can be used to determine the scope of the actual adjustment results to facilitate the adjustment of the actual debugging process. The simulation experiment is done on the experimental platform, and the rationality of the simulation platform and the effectiveness of the path tracking control algorithm are verified by analyzing the debugging results of the actual intelligent car.
\end{abstract}

\section{Introduction}

In recent years, the robot has become a hot research field in mobile robot field at home and abroad, and the path tracking control has become one of the main problems of the automatic guided car. In order to master how the theoretical knowledge is applied in practice and to further improve the performance of intelligent car competition[1-3], this paper studies the development of the platform of intelligent car. The simulation platform can improve the efficiency of the control algorithm and reduce the manpower and material cost, especially for the limited experimental space. Therefore, this paper presents a intelligent car simulation system based on LabVIEW software[4,5]. In order to optimize the control algorithm, different types of runways are often produced in the process of intelligent car speed regulation. This not only wastes a lot of time in making the runway, but also increases the cost of researching intelligent car control algorithms. The simulation platform provides the loading function module of the runway, which can establish the arbitrary runway shape conveniently and quickly, and solves the problems mentioned above.

\section{Introduction of Virtual Instrument}

A virtual instrument is a computer-based tool. The close combination of computer and instrument is an important development direction of the instrument at present. Simply put, there are two ways to accomplish this combination, one is to insert the instrument, the typical example of which is the intelligent instrument computer, which includes increasingly powerful computer functions, and more powerful embedded system equipment functions. Another method is to install the instrument to the computer to the common computer hardware and operating system based on the realization of a variety of instrument functions, virtual instrument mainly refers to this method. In fact, it is based on the demand of data acquisition system for virtual instrumentation. Virtual instrument research mainly involves computer data acquisition and digital signal processing. At present, in this field, the more extensive use of the computer language is LabVIEW.

Like C and BASIC languages, LabVIEW[6,7] is also a common programming system, there is a huge library of tasks to complete the programming. LABVIEW library includes data acquisition, GPIB, serial control, data analysis, data display and data storage and so on. LabVIEW also has traditional debugging tools such as setting breakpoints, animating display data and subroutine results, single stepping, etc. to facilitate the debugging process. LabVIEW is a graphical programming language that 
uses icons instead of text to create an application. Traditional text-based programming language statements determine the order of execution of the program according to the instruction sequence, and then use the LabVIEW data flow programming, data flow diagram between the block diagram to determine the VI and the function of the order of execution. LabVIEW provides a variety of controls that look and feel similar to traditional instruments such as oscilloscopes and multimeters, and can be used to easily create a user interface. The user interface of LabVIEW is called the front panel. It uses icons and connections to programmatically control front panel objects. This is the graphics source code, also known as G code. Similar to the way flow charts LabVIEW graphical source code, it is also known as the block diagram code.

\section{The Design of Intelligent Car Experimental Platform Based on LabVIEW}

The Module of Runway Loading. As the design is completely off-line simulation platform, so the intelligent car tracking process is not through the external sensor input signal. The idea of the development of the intelligent car platform is to determine the position of the intelligent car through the pre-conceived runway and take the uniform point coordinates on the runway. Therefore, the loading of the runway is arbitrary, and the shape of the runway can be changed by arbitrarily changing the coordinate value. This provides a visual simulation environment for various runway PID debugging. Runway loading module display interface is shown in Fig. 1.

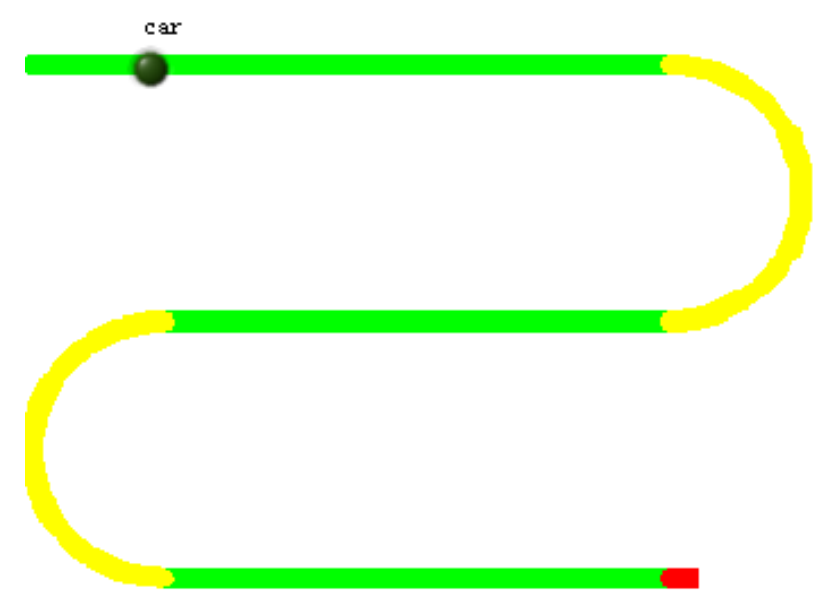

Figure 1. Runway loading module

The Control Module. At present, it is often used to test the parameters of PID control algorithm[8-10] of intelligent car. Due to the lack of the necessary theoretical guidance and lack of experience, we often waste a lot of time in the adjustment process. Because of the need for intelligent cars repeatedly debugging, which often causes damage to the hardware and increases costs. Therefore, it is very important to set up the correct method to solve these problems. It is worth to study the actual adjustment process on the off-line simulation platform.

In the off-line simulation platform, different speed values are set on the runway of different shapes, and the PID value is adjusted by the conversion process of the speed value to determine the final running speed of the intelligent car. In the simulation process, P, I, D three values of the entire simulation process is the core of the process, when the PID value changes, the intelligent car running speed changes will be different, the ideal set of speed and actual value The difference between the speed values will be different. In the LabVIEW environment, the speed of the intelligent car is to adjust the actual speed based on ideal speed, which is got by P, I, D input value by using the PID module. Through the adjustment process of the PID module, Speed value is assigned to the corresponding position in the intelligent car, which completes the speed of the debugging process. This is the purpose of selecting the debugging process of off-line simulation intelligent car. The process of repeated and troublesome PID 
debugging is carried out on off-line simulation platform, and the hardware is replaced by software to save manpower and material resources. PID toolkit is shown in Fig. 2.

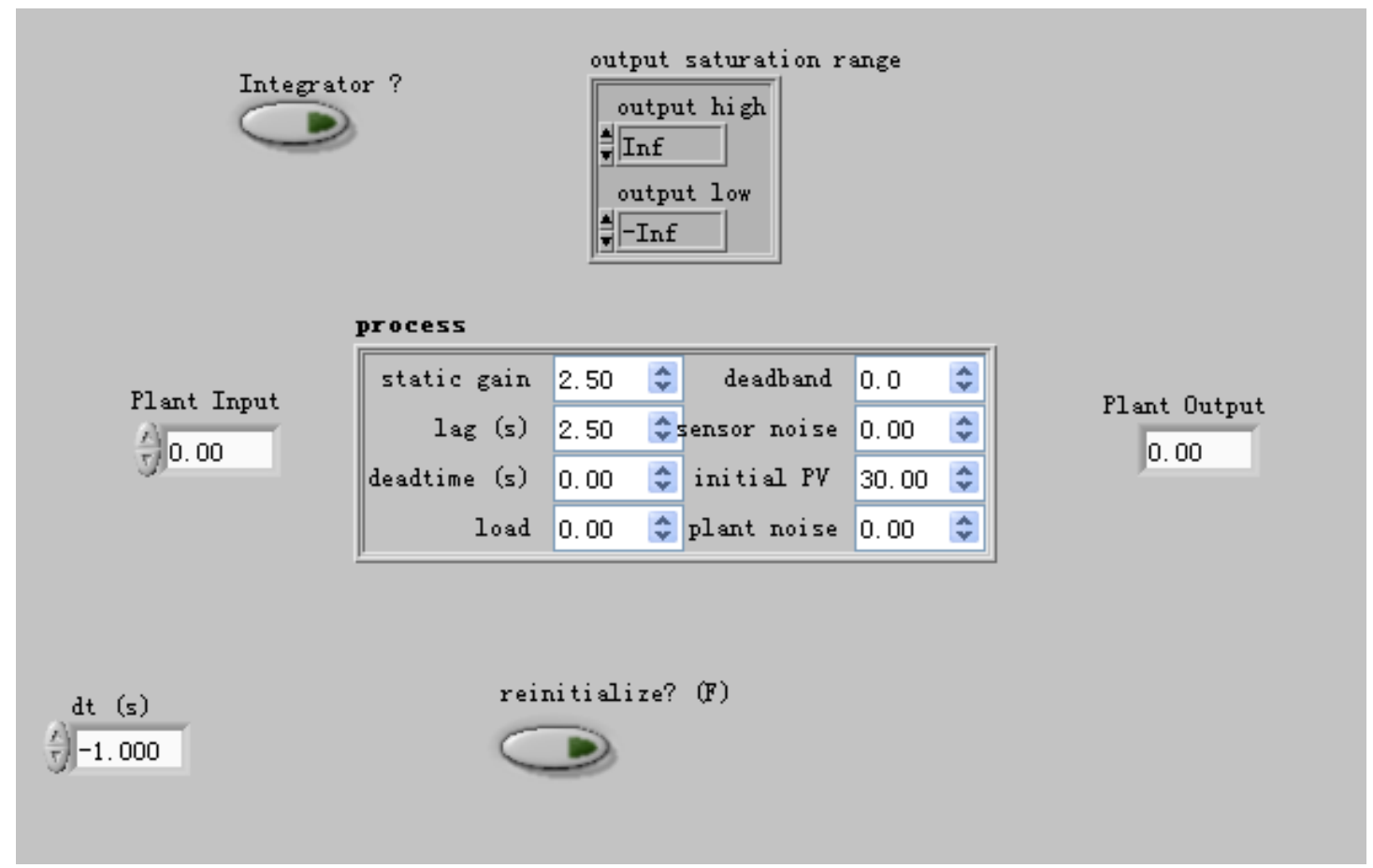

Figure 2. PID toolkit

The Module of Real-time Monitoring. In the intelligent car simulation of the entire visual interface, in addition to intelligent car running along the runway, the most important is the speed of the real-time monitoring of the process of change, the ideal speed of the set process, the actual PID regulation of the speed of change process. The idea of real-time monitoring is to show the change of velocity with time and runway, and to compare the ideal speed with the actual speed. It is convenient to determine the accuracy and validity of PID. In order to make the PID debugging results more accurately expressed, the simulation software will be the ideal speed and the actual speed value of the deviation between the value of real-time monitoring, through the deviation of the curve to a more intuitive entry into the PID worth debugging. Real-time monitoring is shown in Fig. 3.

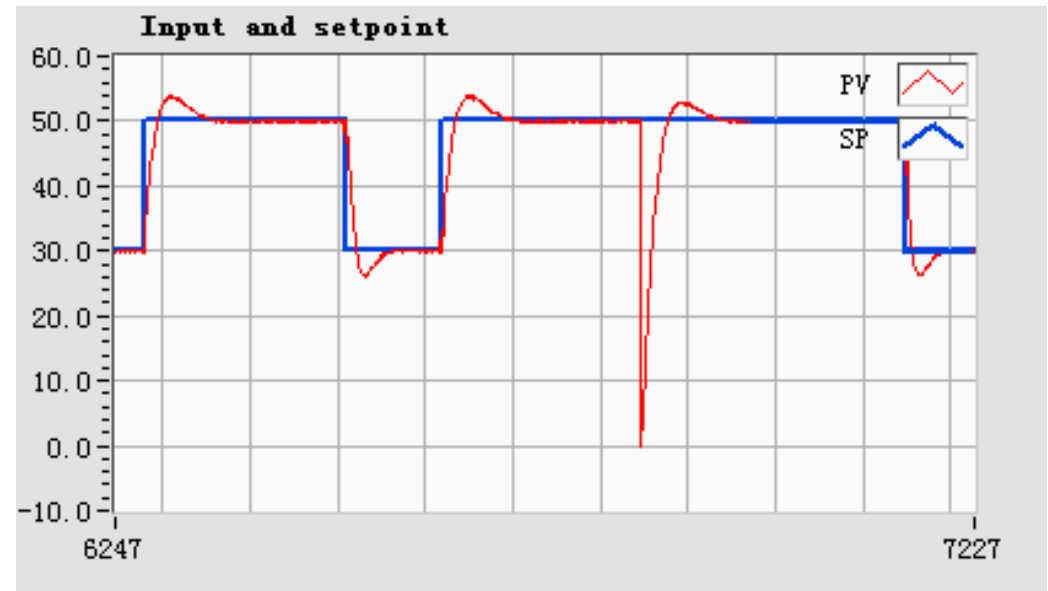

Figure 3. Curve of real-time monitoring

The control panel of the experimental platform is shown in Fig. 4. 


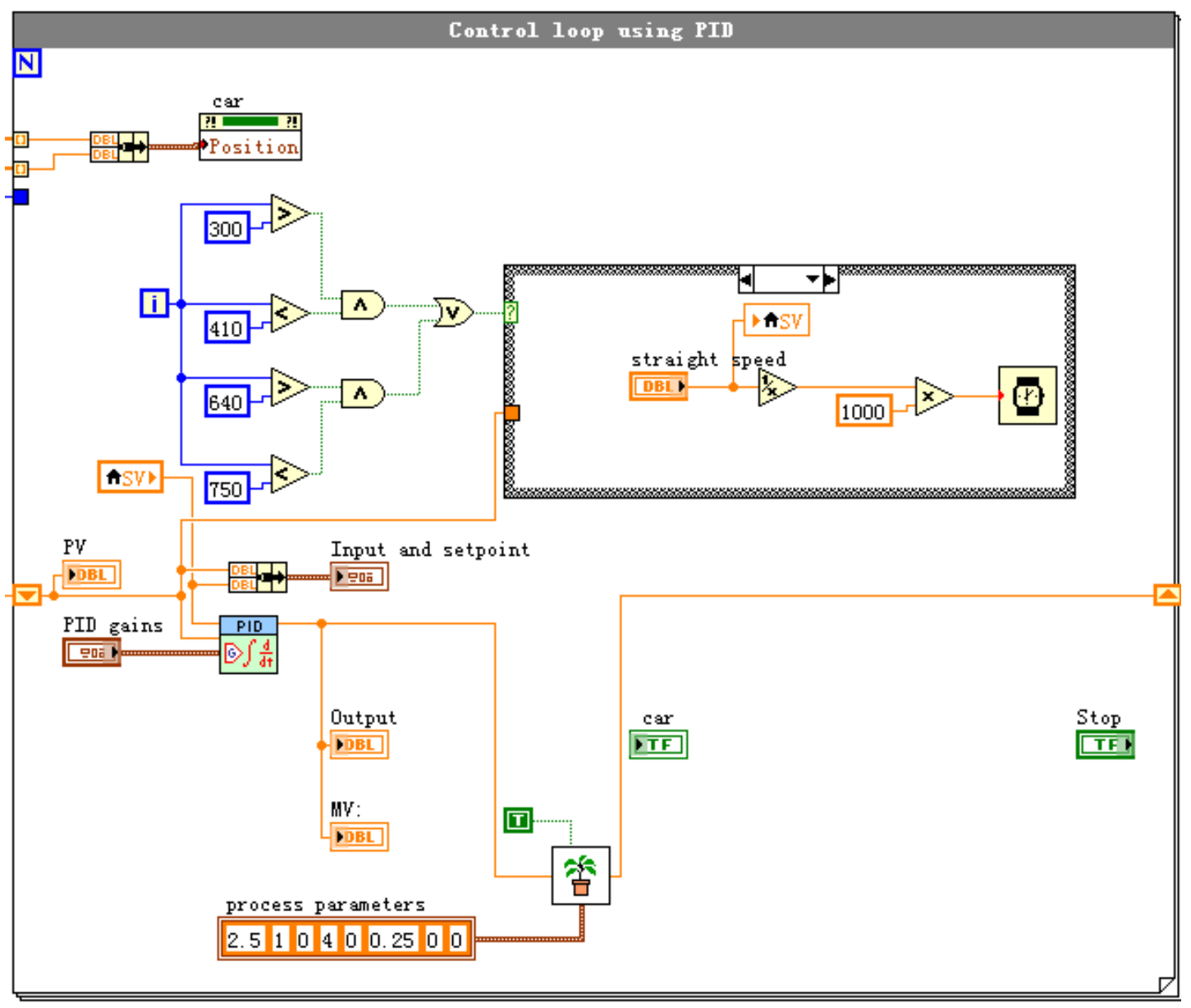

Figure 4. The control panel of the experimental platform

\section{Conclusions}

In this paper, the intelligent car simulation system based on LabVIEW software is put forward. In the establishment of the real platform, the PID parameters tuning, runway loading module, real-time monitoring module and simulation control module will be emphasized. The runway loading module can easily load different runways. The real-time monitoring module can make the user see the running status of the intelligent car so that they can make the intelligent car speed running time more accurate simulation. Users use the simulation control module can control the software running process. Therefore, the platform based on LabVIEW can be well used for simulation of intelligent cars.

\section{Acknowledgements}

This work was supported in part by Liaoning Province Education Science "13th Five-Year Plan" project under Grant No. JG16DB321, JG16DB523, "12th Five-Year Plan" project under Grant No. JG15DB289 and JG15DB274 and Science Research General Project of Liaoning Education Department under Grant L2014530.

\section{References}

[1] Guo L, Ge P S, Yue M, et al. Lane Changing Trajectory Planning and Tracking Controller Design for Intelligent Vehicle Running on Curved Road[J]. Mathematical Problems in Engineering, 2014, 2014(8):1-9.

[2] Yang D, Liu T, Hu C. Design of an intelligent car for searching track and avoiding obstacles[J]. International Journal on Smart Sensing \& Intelligent Systems, 2015, 8(2):1354-1378. 
[3] Shah S, Patel S, Narula H. Intelligent Vehicle Theft Control using Embedded System[J]. International Journal of Computer Applications, 2014, 107(19):34-37.

[4] Ponce H, Ponce P, Molina A. The development of an artificial organic networks toolkit for LabVIEW[J]. Journal of Computational Chemistry, 2015, 36(7):478-92.

[5] Zhu W, Liu J, Yang H, et al. Design of High Precision Temperature Measurement System based on Labview[J]. International Journal of Advanced Computer Science \& Applications, 2015, 6(6):193.

[6] Duan J, Shi H, Liu D, et al. Square Root Cubature Kalman Filter-Kalman Filter Algorithm for Intelligent Vehicle Position Estimate[J]. Procedia Engineering, 2016, 137:267-276.

[7] Du M, Mei T, Chen J, et al. RRT-based motion planning algorithm for intelligent vehicle in complex environments[J]. Jiqiren/robot, 2015, 37(4):443-450.

[8] Sahu R K, Panda S, Pradhan P C. Design and analysis of hybrid firefly algorithm-pattern search based fuzzy PID controller for LFC of multi area power systems[J]. International Journal of Electrical Power \& Energy Systems, 2015, 69:200-212.

[9] Liem D T, Truong D Q, Ahn K K. A torque estimator using online tuning grey fuzzy PID for applications to torque-sensorless control of DC motors[J]. Mechatronics, 2015, 26:45-63.

[10] Kolarik G L. Coordination of PSS and PID Controller for Power System Stability Enhancement Overview[J]. Aba Journal, 2015, 8(2):142. 